\title{
Frailty Testing Pilot Study: Pros and Pitfalls
}

\author{
Taylor Adlamª, Elizabeth Ulrich ${ }^{\mathrm{a}}$, Missy Kent ${ }^{\mathrm{b}}$, \\ Lauren Malinzak ${ }^{\text {b, } c}$
}

\begin{abstract}
Background: Frailty can be defined as an inflammatory state with a loss of physiologic reserve in multiple systems that manifests as a decreased ability to respond to stressors that ultimately leads to an increased risk of adverse outcomes. The aim of this study was to determine the ease of frailty testing in a pre-kidney transplant clinic and the resources required to do so. A secondary goal was to better understand the utility of frailty testing when evaluating potential kidney transplant recipients.
\end{abstract}

Methods: Frailty testing was conducted at a pre-kidney transplant clinic in three phases using Fried's frailty phenotype (shrinking, exhaustion, low physical activity, slowness, and grip strength).

Results: A total of 132 frailty tests were completed on 128 patients. Frail patients had significantly higher rates of shrinking (26\% vs. $8.5 \%, \mathrm{P}<0.05$ ), exhaustion ( $82.6 \%$ vs. $27.6 \%, \mathrm{P}<0.05$ ), low physical activity $(78.2 \%$ vs. $19.0 \%, \mathrm{P}<0.05)$, slow walking $(60.8 \%$ vs. $15.2 \%, \mathrm{P}<0.05)$, and grip strength $(73.9 \%$ vs. $25.7 \%, \mathrm{P}<0.05)$. When comparing the listing of frail and non-frail patients for transplant, a significantly lower proportion of frail patients were listed compared to non-frail patients $(30.4 \%$ vs. $57.6 \%, \mathrm{P}<0.05)$. Frailty testing was most complete when an examiner dedicated to frailty testing performed the testing.

Conclusions: Frailty testing is feasible to complete in a pre-transplant clinic with an appropriate investment in personnel and resources.

Keywords: Clinical research/practice; End-stage renal disease; Frailty testing; Kidney transplant

\section{Introduction}

As the burden of chronic disease increases, the medical community has begun to recognize the need for tools to assess and

Manuscript submitted September 17, 2017, accepted October 3, 2017

a'Wayne State University School of Medicine, Detroit, MI, USA

bDepartment of Transplant, Henry Ford Hospital, Detroit, MI, USA

${ }^{\mathrm{c} C o r r e s p o n d i n g ~ A u t h o r: ~ L a u r e n ~ M a l i n z a k, ~ H e n r y ~ F o r d ~ H o s p i t a l, ~} 2799$ W.

Grand Blvd, Detroit, MI 48202. Email: 1malinz1@hfhs.org

doi: https://doi.org/10.14740/jocmr3203w manage groups of patients. There has been growing research into the components of frailty and its implications on patients and their overall health. In recent years, there has been a specific focus on frailty in patients with end-stage renal disease undergoing renal transplant. Frailty is a multi-faceted concept, but it is universally understood to be a global phenotype that results from reserve losses or physiologic dysregulation [1]. Frailty is considered an inflammatory state associated with an increased vulnerability to adverse outcomes [2].

Frailty scores have been utilized to predict a variety of outcomes. Within the renal transplant population, increased frailty has been associated with delayed graft function, early hospital readmission, increased length of hospital stay, and increased postoperative mortality [2-5]. If the concept of frailty is to be used routinely as a prognostic tool, however, it must be easily applied in a standardized manner. The purpose of this pilot study is to assess the feasibility and utility of incorporating frailty testing into the pre-kidney transplant evaluation process. Aims include identifying the resources necessary to conduct complete frailty testing, evaluating the willingness of the patients to comply with testing, and observing the necessary skill of an examiner conducting frailty testing. A secondary intention included the assessment of frailty testing utility in the pre-kidney transplant evaluation and identification of the resources required to obtain meaningful assessments.

\section{Methods}

\section{Measures}

Frailty testing was completed using the validated Fried's frailty phenotype which uses shrinking, exhaustion, low physical activity, slowness, and grip strength to assess frailty [6]. Each category has a standard set of criteria, and patients are awarded one point if the baseline is not met. Total frailty score is obtained from summing the points in all five categories. A score of 0 is considered not frail. A score of 1 - 2 is considered intermediately frail or pre-frail. A score of 3 - 5 is considered frail.

To determine shrinking, patients were asked to compare their weight 1 year ago to their current weight. If the patient had unintentionally lost 10 or more pounds, then the patient received one point. Shrinking was not completed if the patient did not know their weight 1 year ago.

Exhaustion was assessed by asking the following questions from the CES-D scale: how often in the past week did you feel everything you did was an effort, and how often in the 
past week did you feel you could not get going? If the patient responded three or more times a week for either question, they were awarded one point.

Patients were then asked about frequency of physical activity using the short version of the Minnesota leisure time activity questionnaire. Physical activity was converted to kcals per week using myfitnesspal.com. Men who expended less than 383 kcals per week received one point. Women who expended less than 270 kcals per week received one point.

Slowness was assessed by the time to walk 15 feet. Three trials were performed by the patient, and the time results averaged for one mean score. Patients received one point if they did not meet the time criteria determined by their gender and height. Men who were $173 \mathrm{~cm}$ tall or less and required more than $7 \mathrm{~s}$ to walk 15 feet received one point. Men who were greater than $173 \mathrm{~cm}$ tall and required more than $6 \mathrm{~s}$ to walk 15 feet received one point. Women who were $159 \mathrm{~cm}$ tall or less and required more than $7 \mathrm{~s}$ to walk 15 feet received one point. Women who were greater than $159 \mathrm{~cm}$ and required more than $6 \mathrm{~s}$ to walk 15 feet received one point.

Handgrip strength was measured using a Jamar handheld dynamometer. Three trials were performed by the patient, and the results averaged for one mean score. The cutoff for adequate handgrip strength was determined by the patient's gender and BMI. Men whose BMI was $24 \mathrm{~kg} / \mathrm{m}^{2}$ or less and grip strength was $29 \mathrm{~kg}$ or less received one point. Men whose BMI was between 24.1 and $28 \mathrm{~kg} / \mathrm{m}^{2}$ and grip strength was $30 \mathrm{~kg}$ or less received one point. Men whose BMI was $29 \mathrm{~kg} / \mathrm{m}^{2}$ or greater and grip strength was $32 \mathrm{~kg}$ or less received one point. Women whose BMI was $23 \mathrm{~kg} / \mathrm{m}^{2}$ or less and grip strength was $17 \mathrm{~kg}$ or less received one point. Women whose BMI was 23.1 to $26 \mathrm{~kg} / \mathrm{m}^{2}$ and grip strength was $17.3 \mathrm{~kg}$ or less received one point. Women whose BMI was 26.1 to $29 \mathrm{~kg} / \mathrm{m}^{2}$ and grip strength was $18 \mathrm{~kg}$ or less received one point. Women whose BMI was $30 \mathrm{~kg} / \mathrm{m}^{2}$ or greater and grip strength was $21 \mathrm{~kg}$ or less received one point.

\section{Preparation}

Prior to initiating testing, an institutional review board (IRB) outlining how frailty testing would occur in a pre-kidney transplant clinic was submitted and subsequently approved. Materials needed to initiate frailty testing included a Jamar handheld dynamometer, stopwatch, tape measure and masking tape, and access to myfitnesspal.com. The Jamar handheld dynamometer measured grip strength. The tape measure and masking tape were used to mark a 15-foot course for the slow walking trial, and the stopwatch was used to track time. Myfitnesspal.com was used to calculate the energy expenditure of the patient. Additional data necessary for calculating frailty included the date of testing, age, height, weight, BMI, and gender.

\section{Participants}

Patients were recruited from a pre-kidney transplant clinic as they completed evaluation for a first or second kidney transplant listing. All patients presenting to the kidney pre-trans- plant clinic were eligible for frailty testing. Patients were explicitly told that frailty testing results would not be used to determine eligibility for kidney transplant listing.

\section{Data collection}

Phase one of frailty testing was conducted in summer 2015 by a volunteer medical student. The tester assessed 64 patients. In phase one, patients undergoing pre-kidney transplant evaluation were approached and asked to participate in frailty testing. If patients agreed, they read and signed a consent form prior to participation. As frailty testing proved to pose little to no risk to patients as no falls or injuries occurred during evaluation, it was determined and approved by the IRB that verbal consent to frailty testing would be adequate for future patients. Phase two of frailty testing was conducted in summer 2016 by a volunteer medical student. Only 40 patients were evaluated in this cohort due to dynamometer miscalibration interrupting testing. Phase three of frailty testing was conducted in May 2017 by a volunteer medical assistant, and 28 patients were assessed at a pre-transplant outreach clinic. Testing during this phase was conducted during psychological evaluation appointments that occur during transplant evaluation. This appointment was chosen due to its extended length.

\section{Examiners}

Three examiners were instructed in frailty testing. Education was similar for each tester as they were introduced to the Fried method of testing and familiarized with the frailty testing tools. Responsibilities included consenting the patient, asking about shrinking, exhaustion, and physical activity, timing the 15-foot walk, and operating the Jamar hand-held dynamometer. Examiners experienced a short learning curve but became proficient within five patients. This learning curve did not pose any additional risk to the patient but increased the time to complete testing. Of note, frailty testing was conducted based on examiner availability. Two of the three examiners were volunteer medical students, and the third examiner was a medical assistant who completed frailty testing while fulfilling her clinic responsibilities. With limited resources and a projected short learning curve, the goal was to test at least 100 patients.

\section{Results}

Overall, 132 frailty tests were conducted on 128 patients. One hundred sixteen patients were being evaluated for first transplant, and 12 patients were being evaluated for second transplant. Four patients who were being evaluated for first transplant were tested twice, first in 2015 and subsequently in 2016. In total, five patients refused frailty testing. Three opted out due to time constraints. Two patients could not participate in walking due to immobility.

During phase one in 2015, 64 patients were tested, and testing was $100 \%$ complete. Of these 64 patients, 14 patients 
Table 1. Number of Patients Tested, Completeness of Testing, and Number of Frail Patients in the Three Testing Phases

\begin{tabular}{lllll} 
Cohort & $\begin{array}{l}\text { Patients } \\
\text { tested }\end{array}$ & $\begin{array}{l}\text { Testing } \\
\text { completed }\end{array}$ & Frail & $\begin{array}{l}\text { Inconclusive } \\
\text { for frailty }\end{array}$ \\
\hline 2015 & 64 & $64(100 \%)$ & $14(15.6 \%)$ & $0(0 \%)$ \\
2016 & 40 & $35(87.5 \%)$ & $8(20 \%)$ & $0(0 \%)$ \\
2017 & 28 & $13(46 \%)$ & $1(3.5 \%)$ & $4(14.3 \%)$ \\
\hline
\end{tabular}

(21.8\%) were found to be frail. During phase two in 2016, 40 patients underwent testing, and testing was $87.5 \%$ complete. Testing was incomplete due to miscalibration of the dynamometer. Eight patients $(20 \%)$ were found to be frail. During phase three in 2017, 28 patients were evaluated, and testing was $46 \%$ complete. For this third cohort, shrinking data were missing in 14 of $28(50 \%)$ patients as patients could not report their weight 1 year prior. One patient could not complete the hand grip strength assessment. Only one patient (3.5\%) was found to be frail. Of note, one missing variable did not affect the calculation of frailty if all other testing was complete and only 0 - 1 factors were abnormal. However, if a data point was missing and two other factors were suggestive of frailty, testing was deemed inconclusive. The number of patients tested, completeness of testing, and number of frail patients in the three testing phases are shown in Table 1.

Based on the individual frailty measures of the 128 conclusive frailty tests, frail patients have higher rates of shrinking, exhaustion, low physical activity, slow walking and weak grip strength. Each of these variables independently is significantly higher in the frail patients as compared to the non-frail patients. These results are shown in Table 2.

Of the patients who underwent frailty testing during transplant evaluation, frail patients seemed more likely to be excluded from transplant at the first appointment or die during the evaluation period. However, these differences were not a significant difference between the frail and non-frail groups. Of note, one patient was lost to follow-up during the evaluation process. Although a large group of patients in both the frail and non-frail groups are still waiting to be listed for transplant 6 months to 2 years after their initial evaluation, a significantly lower proportion of frail patients have been listed as compared to non-frail patients. The evaluation status of patients who underwent frailty testing is shown in Table 3.

Of the 67 patients who were listed during follow-up, 25 received a kidney transplant. Two patients died after listing: one was awaiting second transplant and the other had already been on dialysis vintage of 8 years. Of the transplanted patients, 22 were from the non-frail group and three were frail
Table 3. Evaluation Status of Patients Who Underwent Frailty Testing

\begin{tabular}{llll}
\hline After first evaluation & Total (127) & Not frail (104) & Frail (23) \\
\hline Not a candidate & $11(8.6 \%)$ & $7(6.7 \%)$ & $4(17.4 \%)$ \\
Died in evaluation & $4(3.1 \%)$ & $2(1.9 \%)$ & $2(8.6 \%)$ \\
Remains in evaluation & $45(35.4 \%)$ & $35(33.6 \%)$ & $10(43.4 \%)$ \\
Listed & $67(52.7 \%)$ & $60(57.6 \%)$ & $7(30.4 \%)^{*}$ \\
\hline
\end{tabular}

${ }^{*} \mathrm{P}<0.05$ by Chi-squared.

patients. Of the non-frail patients who were transplanted, 11 $(50 \%)$ underwent deceased donor kidney transplant and 11 $(50 \%)$ underwent living donor kidney transplant. All three frail patients underwent living donor kidney transplant. The transplant status of frailty tested patients after listing for transplant is shown in Table 4.

\section{Discussion}

Frailty testing is generalizable and safe in the pre-kidney transplant population. Patients are often willing to comply with testing, and the resources necessary to conduct complete frailty testing can be maintained within the clinic setting. The skills necessary for examiners to obtain meaningful assessments are quickly acquired with minimal guidance. While it may be feasible to complete frailty testing in a busy pre-transplant clinic, caveats to success do exist. Challenges frailty testing faces includes adequate time for testing, equipment service, accurate assessment, and standardized use in the pre-kidney transplant evaluation.

\section{Patients}

Patients were willing to participate in frailty testing. Frailty testing was safe and well received by the patient. The clinicians on the kidney transplant team were blinded to the outcome of the frailty testing, hence the results of the testing should not have influenced the patient's ability to move forward to listing or transplant. Nonetheless, fewer frail patients were listed and no frail patient underwent deceased donor kidney transplant. This could be that frail patients were taking longer to get through the evaluation process, waiting longer on the list for deceased donor kidney transplant based on age and quality of kidney offers. Or this could reflect that frail patients had comorbidities or other barriers to receiving a transplant and were placed status 7 on the transplant list, thus receiving no

Table 2. Prevalence of Frailty Criteria Among Tested Patients

\begin{tabular}{llllll} 
Frailty factors occurrences & Shrinking & Exhaustion & Low physical act & Slow walking & Grip strength \\
\hline Present (total, 128) & $15(11.7 \%)$ & $48(37.5 \%)$ & $38(29.6 \%)$ & $30(23.4 \%)$ & $44(34.3 \%)$ \\
Present in frail (23 patients) & $6(26 \%)^{*}$ & $19(82.6 \%)^{*}$ & $18(78.2 \%)^{*}$ & $14(60.8 \%)^{*}$ & $17(73.9 \%)^{*}$ \\
Present in non-frail (105 patients) & $9(8.5 \%)$ & $29(27.6 \%)$ & $20(19.0 \%)$ & $16(15.2 \%)$ & $27(25.7 \%)$ \\
\hline
\end{tabular}

${ }^{*} \mathrm{P}<0.05$ by Chi-squared. 
Table 4. Transplant Status of Patients Frailty Tested After Listing for Transplant

\begin{tabular}{llll}
\hline After listing & Total (67) & Not frail (60) & Frail (7) \\
\hline Died on list & $2(2.9 \%)$ & $2(3.3 \%)$ & $0(0 \%)$ \\
Transplanted & $25(37.3)$ & $22(36.6 \%)$ & $3(42.8 \%)$ \\
Deceased donor transplant & $11(44 \%)$ & $11(50 \%)$ & $0(0 \%)$ \\
Living donor transplant & $14(56 \%)$ & $11(50 \%)$ & $3(100 \%)$ \\
\hline
\end{tabular}

deceased donor kidney offers. Given the small number of patients and relatively short follow-up compared to the deceased donor kidney transplant wait times, it is difficult to draw any sure conclusions.

Once listed, frail patients experience statistically similar rates of living donor kidney transplant. Frail patients, similar to elderly patients, may benefit from a planned surgery [7]. The good quality organ, lower risk of delayed graft function, potential for lower immunosuppression, and earlier return to baseline function could potentially reduce physiologic stress to the patient at the time of transplant. It may be reasonable to review in the future if frail patients with low physiologic reserve and increased vulnerability to adverse outcomes tolerate live donor kidney transplant better than deceased donor kidney transplant.

\section{Examiners}

During 2015 and 2016 testing, examiners were exclusively dedicated to conducting frailty testing. During 2017 testing, a medical assistant performed frailty testing as an extension of vital signs, but the medical assistant's primary responsibilities in the clinic were to obtain the vital signs and room the patient which included verifying past medical history, past surgical history, family history, allergies, and medications, and conducting frailty testing. A major flaw in this approach was that with limited time, frailty testing was often left incomplete as immediate patient care had to be prioritized. Frailty testing takes approximately $15 \mathrm{~min}$ to complete and requires an additional 10 min to calculate the energy expenditure and record this in the patient's chart. Although time could be cut down marginally if the patients were given the written question to fill out without the testers oral prompting, this may change the accuracy or completeness of the answers. Also, testing the third cohort at a subsequent appointment, rather than the initial assessment may have already screened out those patients that were deemed not a candidate at the initial evaluation appointment. This may account for fewer patient labeled as frail in the third cohort.

When the medical assistant was completing the testing, the testing was complete only $46 \%$ of the time. For example, the medical assistant struggled to assess shrinking when patients were unsure of their prior weights. While examiners should be armed with follow-up questions to help extract this information from the patient or could attempt to obtain weights from prior medical records, an additional time investment would be required. The medical assistant also did not calculate energy expenditure or record the outcomes of the frailty testing in patients' chart. Based on the differences in data collection and analysis, it is evident that frailty testing is best conducted by a person who has dedicated time to complete the frailty testing, calculate the energy expenditure, and record the values in the patient chart. The data collection by a dedicated examiner was most complete and accurate.

\section{Equipment}

Equipment maintenance proved to be a major burden during testing. The dynamometer was the most expensive tool required for frailty testing. During the second year of testing, the project was interrupted as the dynamometer needed to be sent out for re-calibration, which took 2 - 3 weeks. Barriers to measuring accurate hand grip strength could be overcome with a scheduled dynamometer maintenance program and more than one dynamometer available to be used for testing in the case of equipment malfunction.

\section{Calculations}

Frailty phenotype testing requires several calculations. These calculations include energy expenditure, average walking speed, and average grip strength. To calculate energy expenditure, myfitnesspal.com was used rather than the suggested mathematical assessment [6]. These calculations add an additional $10 \mathrm{~min}$ to the total length of frailty testing. One possibility to mitigate the time of calculations and the workload of calculations on the frailty tester includes designing a location in the electronic medical record for frailty information with a preset computer program for calculations.

\section{How to mainstream frailty}

When listing for kidney transplant, clinicians attempt to assess both the patient's health status and perioperative risk. Perioperative frailty can be assessed robustly at first impression within the first minute of meeting the patient in the outpatient setting [8]. This is advantageous because frailty testing (whether for phenotype or index) can be time consuming, cumbersome, require specially trained staff and in the end, is only marginally better than a trained clinician's first impression. The concern is that if left to first impressions, frailty is subjective and clinician dependent. There are over 20 types or combination of frailty testing and indexes reported [9]. There is even a gadget one wears for $20 \mathrm{~s}$ for gait assessment and combined with a questionnaire to predict frailty. May biosensors be the holy grail to simplifying and standardizing frailty testing [10]? Frailty testing, although still being honed to its most efficient and useful form, is objective and should improve transparency when dealing with large populations like those waiting for kidney transplant.

Frailty phenotype testing is a graded scale and the degree of frailty can help guide when pre-habilitation could be useful. Early stages of frailty could benefit from intervention, whereas those with frailty scores of 4 - 5 with metabolic abnormalities 
may have poor response to treatment [11]. Frailty, at its most extreme, could be used to exclude patients from kidney transplant. And for those on the list or in evaluation, annual frailty testing could help to identify patients who are on a downward trajectory: logical to recommend these patients participate in intervention projects prior to transplant. As frail patients are associated with worse outcomes, it will prove important if preconditioning in pre-frail patients improves long-term outcomes and is associated with decreased perioperative morbidity and mortality. Moving forward with frailty testing in a kidney pre-transplant clinic requires applying the knowledge of a frailty score to patient care. Frailty testing seems to add some stratification of risk to the patients who are coming forward for kidney transplant. This stratification can be used to determine patients eligible for pre-conditioning, and patients at increased risk of adverse outcomes.

Cost needs consideration also. Frailty phenotype testing requires trained personnel. Should this examiner be a nurse, medical assistant, or volunteer? Or would centers rather invest in a reliable gadget that will calculate frailty for them in $20 \mathrm{~s}$, which may still require a trained person and integration of the information into the patient's EMR. In kidney transplant, where outcomes matter, centers have flocked to using frailty to predict outcomes. They have considered this a worthwhile investment for now. Hospital costs are well-recognized as being higher in in-patients with frailty $[12,13]$. Prehabilitation could shift the burden of increasing fitness to the patient before transplant, with attrition from fitness programs of $20-67 \%$. Maybe this is asking something that patients cannot complete. Prehabilitation also comes at the unknown cost of time and money while awaiting kidney transplant [14]. Although frailty, based on functional status and ability to perform ADLs, is used as an adjuster to nursing home costs. In the DGR model, frailty, despite its association with higher LOS and discharge to skilled or assistedliving facility, does not receive a higher reimbursement [15].

\section{Conclusion}

In conclusion, frailty testing can be done well if several commitments are made, such as an up-front investment in the tools to complete the testing and investing in the personnel for the time it takes to complete frailty testing. If frailty testing is to be utilized as a useful tool in assessing the pre-kidney transplant population and managing subsequent patient care, it must be easily quantified in an objective standardized manner at minimal cost.

\section{Grant Support}

No grant support.

\section{Author Contributions}

Taylor Adlam and Elizabeth Ulrich: study design, acquisition of data, drafting and revision, final approval. Missy Kent: con- ception and design. Lauren Malinzak: conception and design, analysis of data, drafting and revision, final approval.

\section{References}

1. Rockwood K, Song X, MacKnight C, Bergman H, Hogan DB, McDowell I, Mitnitski A. A global clinical measure of fitness and frailty in elderly people. CMAJ. 2005;173(5):489-495.

2. Garonzik-Wang JM, Govindan P, Grinnan JW, Liu M, Ali HM, Chakraborty A, Jain V, et al. Frailty and delayed graft function in kidney transplant recipients. Arch Surg. 2012;147(2):190-193.

3. McAdams-DeMarco MA, Law A, Salter ML, Chow E, Grams M, Walston J, Segev DL. Frailty and early hospital readmission after kidney transplantation. Am J Transplant. 2013;13(8):2091-2095.

4. McAdams-DeMarco MA, King EA, Luo X, Haugen C, DiBrito S, Shaffer A, Kucirka LM, et al. Frailty, length of stay, and mortality in kidney transplant recipients: a national registry and prospective cohort study. Ann Surg. 2017;266(6):1084-1090.

5. McAdams-DeMarco MA, Law A, King E, Orandi B, Salter M, Gupta N, Chow E, et al. Frailty and mortality in kidney transplant recipients. Am J Transplant. 2015;15(1):149-154.

6. Fried LP, Tangen CM, Walston J, Newman AB, Hirsch C, Gottdiener J, Seeman T, et al. Frailty in older adults: evidence for a phenotype. J Gerontol A Biol Sci Med Sci. 2001;56(3):M146-156.

7. Danovitch G, Savransky E. Challenges in the counseling and management of older kidney transplant candidates. Am J Kidney Dis. 2006;47(4 Suppl 2):S86-97.

8. O'Neill BR, Batterham AM, Hollingsworth AC, Durrand JW, Danjoux GR. Do first impressions count? Frailty judged by initial clinical impression predicts mediumterm mortality in vascular surgical patients. Anaesthesia. 2016;71(6):684-691.

9. de Vries NM, Staal JB, van Ravensberg CD, Hobbelen JS, Olde Rikkert MG, Nijhuis-van der Sanden MW. Outcome instruments to measure frailty: a systematic review. Ageing Res Rev. 2011;10(1):104-114.

10. Toosizadeh N, Joseph B, Heusser MR, Orouji Jokar T, Mohler J, Phelan HA, Najafi B. Assessing Upper-Extremity Motion: An Innovative, Objective Method to Identify Frailty in Older Bed-Bound Trauma Patients. J Am Coll Surg. 2016;223(2):240-248.

11. Pacala, JT, Sullivan GM. Geriatrics review syllabus: a core curriculum in geriatric medicine, 7 th edition. New York: American Geriatric Society. 2010.

12. Shaked A, Lai J. Liver Transplantation in the High MELD Era, Presented at: The Liver Meeting; Nov. 11-15, 2016; Boston.

13. Bock JO, Konig HH, Brenner H, Haefeli WE, Quinzler $\mathrm{R}$, Matschinger H, Saum KU, et al. Associations of frailty with health care costs--results of the ESTHER cohort study. BMC Health Serv Res. 2016;16:128.

14. Cheng XS, Myers JN, Chertow GM, Rabkin R, Chan KN, 
Chen Y, Tan JC. Prehabilitation for kidney transplant candidates: Is it time? Clin Transplant. 2017;31(8):e13020.

15. Makary MA, Segev DL, Pronovost PJ, Syin D, Bandeen-
Roche K, Patel P, Takenaga R, et al. Frailty as a predictor of surgical outcomes in older patients. J Am Coll Surg. 2010;210(6):901-908. 